\title{
Beiträge zur Morphologie und Entwicklungsgeschichte der Blätter einiger Palmen und Cyclanthaceen.
}

Von Max Hirmer.

(Mit 10 Abbildungen im Text.)

Die Entwicklungsgeschichte der Palmblätter ist mehrfach untersucht worden. Die älteren Arbeiten, wie die von Mohl, Hofmeister und Goebel, die nur auf den Untersuchungen an sehr wenig Material fußen, können nicht als abschließend bezeichnet werden, die übrigen eingehenderen Arbeiten von Eichler, Naumann und Deinega gehen auseinander gerade in den Punkten, deren Nichterledigung als Lücken bereits in den älteren Arbeiten empfunden wurde. Zweck vorliegender Arbeit ist, an der Hand moderner Arbeitsmethoden, insbesondere der Mikrotomtechnik, die noch strittigen Punkte einer neuen Untersuchung zu unterziehen. Es handelt sich dabei um folgende zwei Fragen: 1. die Bildung der Blattfläche überhaupt, 2. das Zustandekommen der bei Phoenix vorhandenen Haut an der Blattoberseite.

Die Bildung der Blattfläche im allgemeinen betreffend, so stellt die Mehrzahl der genannten Autoren Faltung der ursprünglich einfachen Blattanlage fest, eine Entwicklungsart, die übrigens nicht auf die Palmen beschränkt ist, sondern typisch ist für großspreitige Blätter überhaupt.

Die Amaryllidee Curculigo diene als Beispiel (Fig. 1). Die anfangs flächige Blattanlage wölbt sich bald auf der Blattunterseite, bald auf der Blattoberseite in alternierenden Strecken vor, wobei der äußerste Blattrand nicht in die Faltung mit einbezogen wird. Im Laufe der Entwicklung greifen die Falten sowohl auf der Blattoberals auf der Blattunterseite tiefer ein. Die beigegebenen Figuren dürften besser als Worte den Vorgang klar machen. Es entsprechen Blattober- und Unterseite des fertigen Blattes denen des jungen noch nicht gefalteten Blattes.

Das wäre nicht der Fall, wenn die große Blattfläche, wie sie die Palmen aufweisen, nicht durch. Faltung, wie sie eben für Curculigo 
beschrieben wurde, zustande käme, sondern durch Spaltung der Emhryonalanlage des Blattes. Mohl für. Cocos und später Naumann auch für Phoenix u. a. glauben derartiges beobachtet zu haben.

Dabei käme die Vergrößerung der Blattfläche zustande durch regelmäßig über einzelne Partien verteilte Wulstbildung an der Blattunterseite, mit der an den Stellen, wo immer je zwei Wülste zusammenstoßen, eine Spaltung des Blattgewebes verbunden wäre. Mit diesen Spalten sollen weitere, gegen die Blattoberseite gerichtete Spalten abwechseln, die ihrerseits entweder bis zum ursprünglichen Blattrand, wie bei der Mehrzahl der Fälle, oder wie bei Phoenix bis nicht ganz

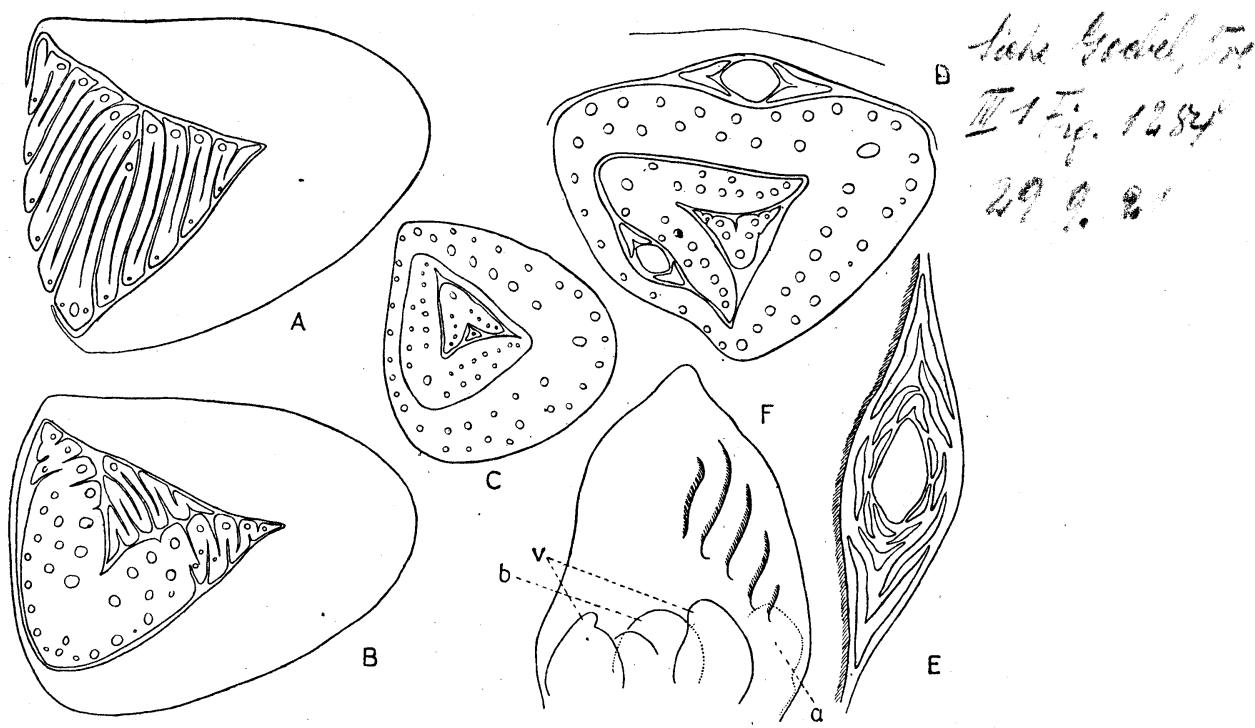

Fig. 1. Curculigo recurvata. $A-C$ Querschnitte durch eine junge Sproßanlage, in verschiedener Höhe. $D$ Querschnitt durch eine weitere junge Sproßanlage mit zwei Infloreszenzsprossen. $E$ Querschnitt durch einen Infloreszenzsproß. $F$ junges Laubblatt frei präpariert. $a=$ nächst jüngeres Blatt; $b=$ Vegetationspunkt des Infloreszenzsprosses; $v=$ dessen beide freie Vorblätter.

soweit durchgreifen würden. Die Oberhaut des fertigen Blattes entspräche demnach der Epidermis des noch ungespaltenen Blattes plus der in gleichmäßigen Abständen eingeschalteten sekundären Epidermis, die als solche erst nach der Spaltung zutage tritt und entwicklungsgeschichtlich aus dem Grundgewebe des Blattes hervorgeht.

Die Annahme einer derartigen Entwicklung des Palmblattes hat etwas Bestechendes in Fällen wie bei Phoenix, wo auf gewissen Stadien die Tatsache der Faltung verschleiert ist. Bekanntlich zeigt ein Querschnitt durch ein jüngeres Phoenixblatt eines drei- oder mehrjährigen Individuums dasselbe ähnlich gefaltet wie bei Curculigo beschrieben 
wurde; vor die Falten aber legt sich ein mit Leitbündeln versehenes zusammenhängendes Band aus weitlumigen, inhaltsarmen Zellen, das sich bald ohne Unterbrechung, bald im Bereich der Mitte jeweils aussetzend, über das ganze Blatt hinzieht. Dieses Gebilde, im allgemeinen in den oben aufgeführten Arbeiten als „Haut" kurz benannt, würde nach der Auffassung von Mohl und Naumann, wie bereits oben angedeutet, zustandekommen dadurch, daß die in den meisten Fällen bis zum ursprünglichen und oberen Blattrand durchsetzenden Spalten ihn hier nicht ganz erreichen. Das von den Spalten nicht berührte Band würde durch Degeneration der Zellen vom übrigen Blattgewebe losgetrennt, die freien, in der Mitte der Aufwölbung gespaltenen Partien der Blattunterseite würden die Teilfiedern des Blattes liefern.

Es sei vorweggenommen, daß die hier besprochene und von Mohl und Naumann angenommene Spaltung nicht nachweisbar ist. Vielmehr liegt auch bei dem am schwersten zu-verstehenden Entwicklungsgang des Blattes von Phoenix, wie auch in den übrigen Fällen, Faltung vor.

\section{Cocos.}

$\mathrm{Da} B$ hier die Anlage der Fiedern durch Faltung vor sich geht und nicht, wie Mohl annimmt, .durch Spaltung, ist bereits festgestellt durch die Arbeiten von Eichler und Deinega.

Zeichnungen nach eigenen Präparaten des Verfassers mögen kurz $\mathrm{zu}$ weiterem Beweis angeführt werden (Fig. 2).

Die gefalteten Partien des Blattes sind zwei relativ schmale Streifen rechts und links der ziemlich mächtig entwickelten Blattrachis. Die Ebenen der beiden Faltungszonen bilden zueinander ungefähr einen rechten Winkel. Der Verlauf der Falten selbst ist nahezu horizontal. Die Faltung geht vor sich, indem sich erst die Blattoberseite an den entsprechenden Partien leicht aufwölbt, etwas später werden die Aufbiegungen auch auf der Blattunterseite bemerkbar, bis schließlich noch ältere Stadien das typische Faltungsbild zeigen. Die Leitbündelanordnung betreffend, so ist der jeweils kräftigste Strang an der Oberkante der Falten, die seitlichen Partien und die Unterkanten der Falten sind von erheblich schwächeren Leitbündeln durchzogen.

Die Auflösung des Blattes in die einzelnen Fiedern erfolgt, wie bereits von Eichler festgestellt wurde und hier der Vollständigkeit halber nur kurz wiederholt werden soll, durch Auflockerung und schließliche Verschleimung des Gewebes an den Unterkanten der Falten. An den Segmenträndern entsteht sekundär wieder eine die übrige Oberhaut fortsetzende Epidermis. 


\section{Phoenix.}

Es ist bekannt, daß, wie auch bei Cocos, auch bei dieser Gattung die Blattspreiten von ein- und zweijährigen Pflanzen ungeteilt sind: Querschnitte durch junge Stadien zeigen das Blatt in der Knospenlage mehrfach gefaltet (Fig. 3). Dabei setzt die erste Aufwölbung der Faltung wieder auf der Blattoberseite ein.

Querschnitte durch etwas ältere Blätter (Fig. 4C-E) zeigen Wucherungen einzelner Epidermiszellen der oberen Faltenkanten und nachfolgende Degeneration des Wucherungsproduktes, wobei der Degenerationsprozeß mehr oder weniger tief in das Gewebe der Faltenkanten eingreifen kann, niemals jedoch so tief, daß er bis zu dem Leitbündel vordränge.

Das ist zum Teil der Fall bei Blättern etwas älterer Pflanzen. Derartige Exemplare zeigen ihre Blattfläche im großen und ganzen

Fig. 2.

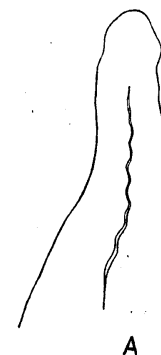

$A$

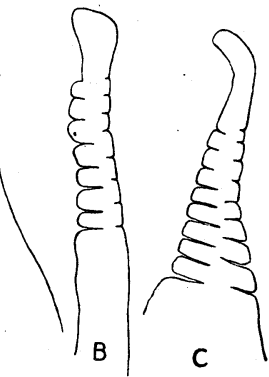

Fig. 3.

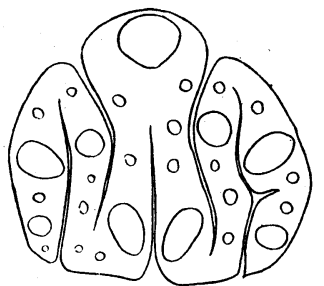

Fig. 2. Coccos spec. Längsschnitte durch die Faltungszone verschiedener alter Blätter. $A$ beide Faltungszonen getroffen; Beginn der Faltung auf der Blattoberseite. $B$ und $C$ nur je eine Faltungszone getroffen.

Fig. 3. Phoenix dactylifera. Querschnitt durch ein junges Blatt einer etwas mehr als einjährigen Pflanze.

noch ungeteilt, nur die äußersten Falten jeder Blatthälfte sind bereits getrennt und als Fiedern der übrigen zusammenhängenden Blattlamina angefügt. Die Auflösung in Fiedern geht vor sich, indem das bereits bei den Blättern jüngster Pflanzen festgestellte Absterben der Zellen der äußersten oberen Faltenecken bis über das Leitbündel hinaus um sich greift, während durch interkalares Wachstum der entsprechenden Teile der Blattmittelrippe die einzelnen Falten auseinandergeschoben werden.

Damit ist im wesentlichen der Mechanismus festgestellt, der die Zerteilung der Blätter der über 3 Jahre alten Pflanzen ermöglicht. Was hier neu hinzutritt ist die bereits eingangs erwähnte Bildung einer 
Haut, die mehr oder minder zusammenhängend sich über die junge noch gefaltete Blattspreite hinzieht. Ihr Zustandekommen wird auch ermöglicht, wenn wir die $\mathrm{Naumannsche} \mathrm{Hypothese} \mathrm{nicht} \mathrm{annehmen,}$ durch folgende Vorgänge, deren Verwirklichung Goebel in Frage zieht: durch Verwachsung einer von der Blattbasis her sich entwickelnden Schuppe, die mit den oberen Kanten der Falten verschmilzt oder durch Verwachsung der Falten mit dem eingeschlagenen Blattrand oder schließlich durch innige Verwachsung der oberen Teile der Blattfalten, eine Möglichkeit, die, wie ihre Aufführung an erster Stelle beweist, dem genannten Autor am wahrscheinlichsten erscheint, wie auch am aufgeführten Ort die Haut als ein nur sekundäres Produkt, die Gliederung der Blattlamina aber als ursprünglich mit der der anderen Palmen übereinstimmend angenommen wird.

Eichler stellt gleichfalls im allgemeinen Faltung fest. Dabei sollen im unteren Teil der Spreite die Oberkanten der Falten frei, im oberen Spreitenteil verschmolzen sein.

Auch De in ega spricht von Faltung; er unterscheidet am jungen Blatt folgende Partien: in der Mitte die Rippe, anschließend links und rechts den gefalteten Teil der Blattspreite, $\mathrm{zu}$ äußerst den ungefalteten Blattrand und zwischen ihm und der gefalteten Blattspreite eine Zone, die selbst nicht mehr gefaltet ist, in die sich aber die anschließenden Falten taschenartig fortsetzen. Diese Zone allein soll im weiteren Entwicklungsverlauf eine Verbreiterung erfahren, während die eigentliche und ursprünglich gefaltete Zone im Wachstum stillstände.

Damit ist den vier oben angeführten Entwicklungsmöglichkeiten eine fünfte zugesellt; viel Wahrscheinlichkeit indes besitzt sie nicht. Denn es muß von vornherein als unwahrscheinlich angesehen werden, daß das Wachstum eines großen Blattes, wie das von Phoenix, nicht innerhalb der Partien vor sich geht, die entsprechend ihrer Faltung hierzu die beste Möglichkeit bieten und innerhalb deren bei den übrigen Vertretern der Familie das Wachstum auch vornehmlich erfolgt, sondern daß es verschoben sei auf eine ursprünglich sehr schmale Zone, die erstens als solche überhaupt nur dadurch charakterisiert ist, daß Faltungszone und Randzone sich hier berühren, während keinerlei anatomische Unterschiede sie auszeichnen, die zweitens aber als dem Blattrand benachbart, von vornherein wenig geeignet ist, eigentliche Trägerin des Wachstums zu sein. Denn gerade in der Randgegend hat bereits bei sehr jungen Stadien das Gewebe seinen embryonalen Charakter verloren. 
Zur Kritik der übrigen angeführten Theorien, so ist es unwahrscheinlich, daß ein Gebilde, das, wie das Phoenixblatt, sich durch nichts wesentlich unterscheidet von den Blättern verwandter Fiederpalmen, entwicklungsgeschichtlich völlig anders zustandekommen soll als diese. Darauf hat ja schon Goebel mit Nachdruck hingewiesen. Dann auch muß dagegen sprechen die hierzu nötige Annahme, daß Jugend- und Folgeform eines Organs in ihrer Entstehung prinzipielle Unterschiede aufweisen sollen; dies gilt besonders für die $\mathrm{N}$ a u mann'sche Theorie. Denn für. das Jugendblatt von Phoenix ist, wie aus den beigefügten Figuren nach Mikrotomschnitten hervorgeht, die auch für die übrigen Palmen charakteristische Faltung nicht mehr von der Hand zu weisen.

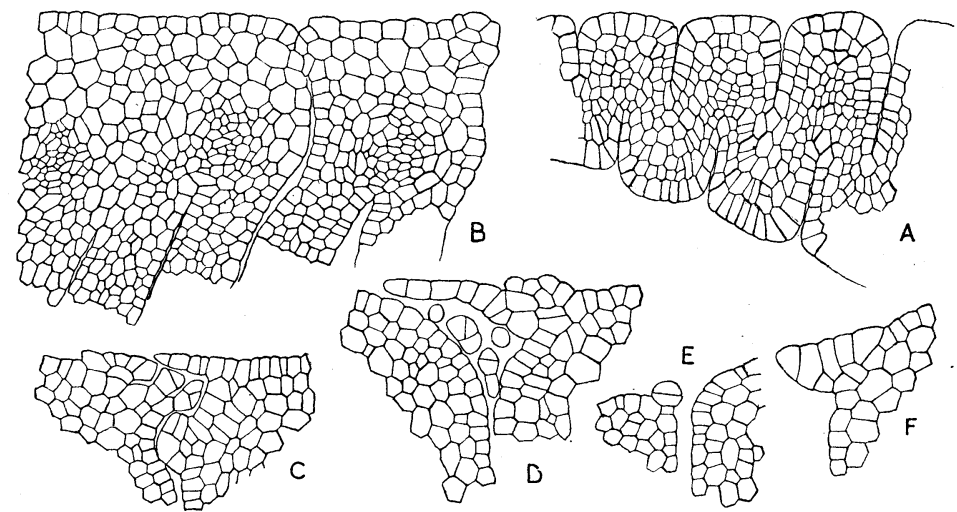

Fig. 4. Phoenix dactylifera. $A$ und $B$ Querschnitte durch Blätter eines fünfjährigen Individuums. $A$ Querschnitt durch die Faltungszone eines sehr jungen Blattes. $B$ Querschnitt durch die Faltungszone eines etwas älteren Blattes. $C-E$ Querschnitte durch die Faltenkanten junger Blätter von 1 und 2 Jahre alten Pflanzen. $F$ Querschnitt durch eine Faltenkante eines Blattes von Carludovica plicata. $C-F$ : Beachte die Wucherungen der Epidermis.

Was nun die Entwicklungsgeschichte der Blätter älterer Phoenixpflanzen betrifft, so kann jetzt auch hierfür die Faltung des Blattes als sicher feststehende Tatsache angenommen werden. Fig. $4 A$ stellt einen Mikrotomschnitt quer durch die Faltungszone eines sehr jungen Blattes dar. Es sind in der gezeichneten Partie deutlich die Aufwölbungen auf der Blattober- und -unterseite sichtbar, Verfolgt man dabei, mit der Epidermis beginnend, die einzelnen Zellschichten, so sieht man deutlich, wie auch sie dem Faltenverlauf folgen. Würde, wie Naumann annimmt, die Faltung sekundär durch Spaltung des Blattgewebes senkrecht zur primären Epidermis zustandekommen, müßte sowohl diese Epidermis als die darunterliegenden Zellschichten des 
Blattgewebes senkrecht eingeschnitten sein, Zellschichten und Faltungslinien könnten keine organische Beziehung aufweisen.

In Stadien, etwas älter als das in Fig. $4 A$ dargestellte, beginnt die Verschmelzung der oberen Faltenkanten des Blattes. Sie ist, wie Fig. $4 B$ zeigt, eine derart innige, daß ein Auseinanderhalten der einzelnen ursprünglich getrennten Faltenkanten unmöglich ist. Dabei wird die
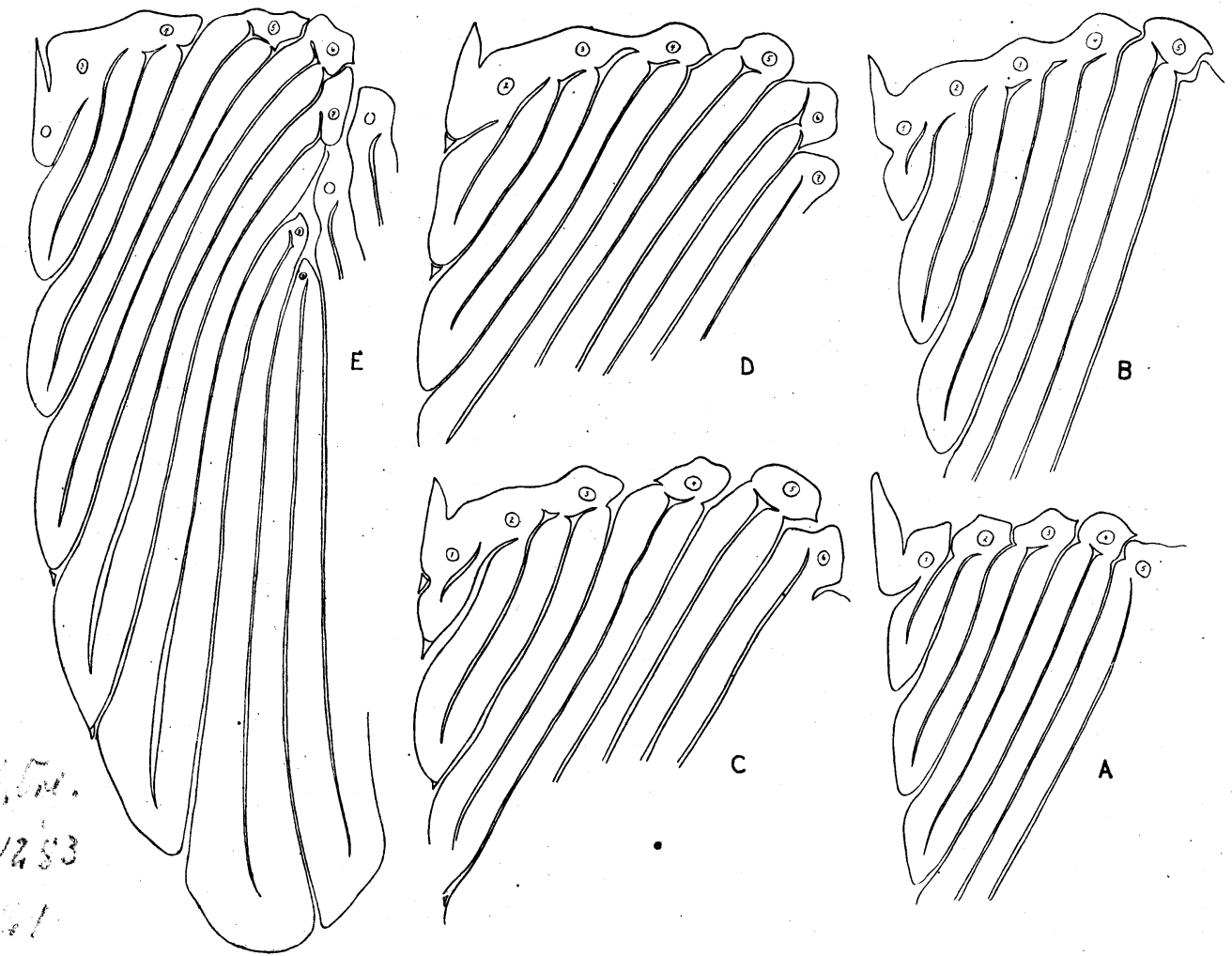

Fig. 5. Phoenix dactylifera. $A-E$ Querschnitte durch ein junges Blatt, in verschiedener Höhe. $E=$ höchster Schnitt; Abstände: $A: B: C: D: E=0,3 \mathrm{~mm}: 0,3$ $\mathrm{mm}: 0,3 \mathrm{~mm}: 0,12 \mathrm{~mm}: 0,6 \mathrm{~mm}$.

Verschmelzung der Kanten noch gefördert durch Volumvergrößerung der einzelnen in Betracht kommenden Zellen, ferner dadurch, daß sekundär einzelne Epidermiszellen, wie das auch für die Blätter ganz junger Phoenixpflanzen bereits angegeben wurde, mehrfach sich teilen und :so die Lücken zwischen den Kanten völlig ausfüllen.

Was nun die Ausdehnung der Verschmelzung der Kanten betrifft, so hat Eichler angegeben, die Oberkanten der oberen Blatthälfte 
würden in ihrer Gesamtheit verschmelzen, die der unteren freibleiben. Nun mag zugegeben werden, daß im allgemeinen die Verschmelzung im oberen Teil der Falten tatsächlich eine allgemeinere ist als im unteren, im besonderen aber fanden sich an den untersuchten Blättern sehr viele Unregelmäßigkeiten. Sowohl insofern, als die Verschmelzung stellenweise sämtliche Faltungskanten einer Blatthälfte erfaßt, um weiter oben und unten die gleichen Kanten frei zu lassen (Fig. 5), als auch insofern, als außer der Verschmelzung sämtlicher Kanten jeder der beiden Blatthälften in manchen Fällen auch die Nachbarkanten der linken und rechten Blatthälfte miteinander verschmelzen (Fig. 6B).

Es kann der erste Fall nicht als direkter Beweis für die Annahme einer Faltung und gegen die Naumann'sche Hypothese angesehen werden. Es wird sich hier bei teilweise vorliegender Verschmelzung der Faltenkanten und teilweisem Freibleiben eben derselben Kanten nur darum handeln, ob man das Freibleiben als primären oder als
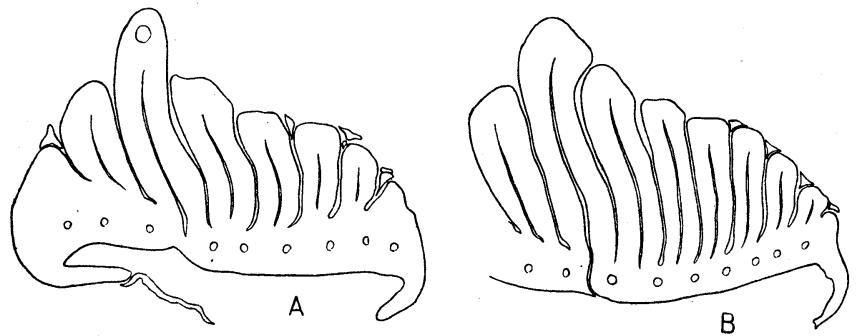

Fig. 6. Phoenix dactylifera. Querschnitt durch ein junges Blatt einer mehrjährigen Pflanze. $A$ höher, $B$ tiefer.

sekundären Zustand auffaßt, mit anderen Worten, ob wir annehmen, daß eine Verschmelzung der, nach der Faltung ursprünglich freien Kanten unterblieben ist, oder, daß eben gerade die sonst sich nur auf die Mittelpartie des Blattgewebes erstreckende Spaltung sich hier bis auf den oberen Blattrand durchgesetzt hat. Was aber, wie auch das obenerwähnte Parallelgehen der einzelnen Zellschichten des Blattes mit den Faltungsbögen, eindeutig für eine wirkliche Faltung des Phoenixblattes und was eindeutig für eine nachträgliche Verschmelzung der Faltungskanten zur Haut spricht, das ist die Tatsache, daß an mehreren Blättern stellenweise die Nachbarkanten der rechten und linken Blatthälfte zu einem zusammenhängenden Ganzen verschmolzen sind, trotzdem ja die Ausbildung beider Blatthälften unabhängig und getrennt durch die größtenteils nichtgefaltete Mittelpartie sich vollzieht. 
Die Tatsache, daß die für die Palmen charakteristische Faltung des Blattes vorkommt auch bei großblätterigen Vertretern anderer Familien, vgl. Curculigo, legte die Frage nahe, 'wie die Blattentwicklung verläuft bei der den Palmen verwandten Familien der

\section{Cyclanthaceen.}

Hier schließt die Gattung

\section{Carludovica}

hinsichtlich ihrer Entwicklungsgeschichte eng an den Palmblatttypus an. Was die Blätter von Carludovica (Fig. 7) den Palmblättern gegenüber auszeichnet ist, daß außer der Betonung der Mittelrippe im Blatt, die ja auch bei den Palmen vorliegt, auch noch bei einzelnen Arten
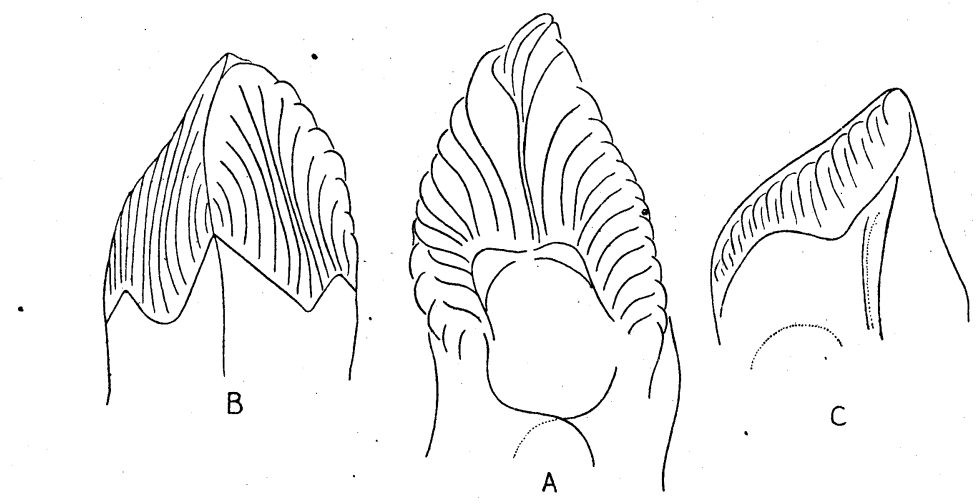

Fig. 7. Carludovica Goebelii. Junge Blattanlage frei präpariert; $A$ von hinten, $B$ von vorne, $C$ von der Seite gesehen.

(Carludovica palmata, C. Goebelii) mehr oder minder stark ausgeprägt, zwei Seitenrippen hervortreten. Entsprechend dieser Tatsache ist der Entwicklungsgang insofern modifiziert, als sich im jungen Blatt an seiner Basis drei vielschichtige Partien bemerkbar machen, die den späteren drei großen Rippen des Blattes entsprechen; die über sie sich hinziehende Partie des Blattes erfährt die Faltung, dabei ist wie auch bei Cuculigo und den Palmen das Mittelstück zwischen den beiden Flanken ungefaltet, während über die zwei aufragenden Seitenrippen die Faltung ununterbrochen fortgeht.

Anders verläuft die Entwicklung von

Cyclanthus.

Sehr früh treten hier bei Cyclanthus bipartitus (Fig. 8 u. 9) kräftig in Erscheinung diejenigen Teile des Blatte̊s, die später die Gabelrippen darstellen. 
Zwischen sie eingeschaltet befindet sich ein kurzes Gewebestück, die mittlere Partie der Blattspreite, und links und rechts von den Gabel-
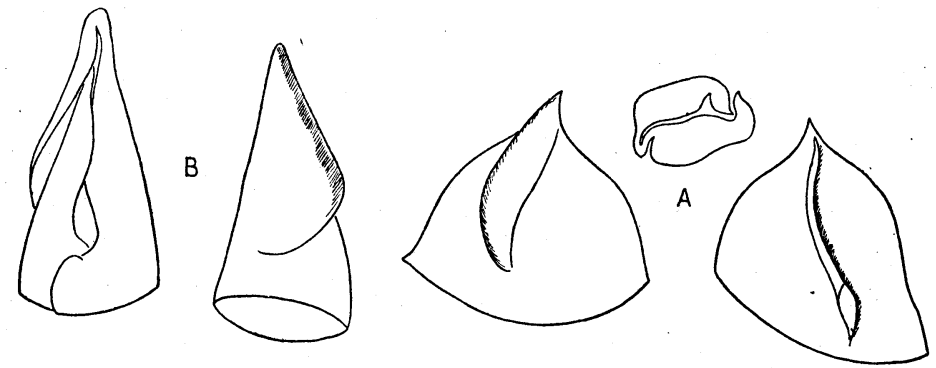

Fig. 8. Cyclanthus bipartitus. Frei präparierte junge Blätter. $A$ sehr junges Stadium; von vorne und von hinten und im Querschnitt. $B$ etwas älteres Stadium; von vorne und von hinten gesehen.

rippen setzt je ein weiteres kurzes Stück an, die beiden Seitenpartien der Blattspreite. Im Laufe der Entwicklung setzt an diesen drei Stellen sehr starkes Wachstum ein; auch hier kommt es bei der Bildung der eigentlichen Blattspreite $\mathrm{zu}$ einer Art Faltung, nur daß es sich um drei Faltungszonen handelt, und daß die Mittelpartie, entsprechend dem gröBeren Spielraum, über den das jüngere Blatt
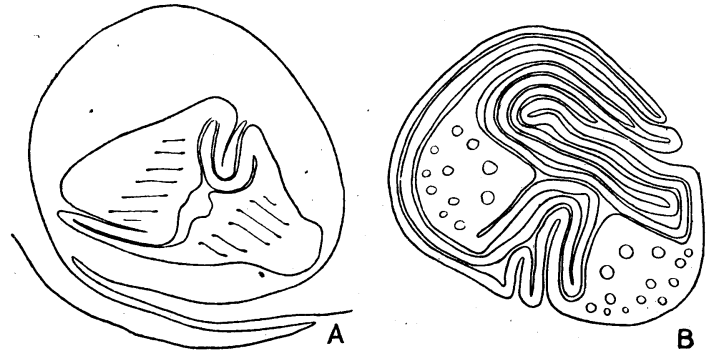

Fig. 9. Cyclanthus bipartitus. Querschnitt durch ein älteres, jedoch noch nicht entfaltetes Blatt. innerhalb des nächst älteren Blattes verfügt, bei ihrem Wachstum eine der Gabelrippen von außen umgreift.

Es ergibt sich die Frage, inwieweit sich die hier geschilderten Blattypen in Beziehung untereinander und in Beziehung zu der für die Monokotyledonen als typisch zu betrachtenden Blattform bringen lassen.

Die Form des eigentlichen Monokotylenblattes (Fig. 10 A) ist repräsentiert in Blättern, bei denen eine Anzahl von gleichmäßig entwickelten Primärnerven einander mehr oder minder parallel verlaufend die Blattspreite durchziehen; eine besonders kräftig entwickelte Mittelrippe, gebildet durch Zusammenfassen der mittleren Leitbündelstränge, ist nicht vorhanden. 
Davon lassen sich unmittelbar ableiten: Formen mit ausgebildeter Mittelrippe: Die mittleren Blattnerven der linken und rechten Blatthälfte nähern sich im unteren Teile der Blattspreite sehr stark dem Mediannerven (Fig. 10 B).

Curculigo von den hier untersuchten Formen vertreten den Typus. Ihm zuzurechnen sind auch die Blätter der Fiederpalmen, nur daß hier
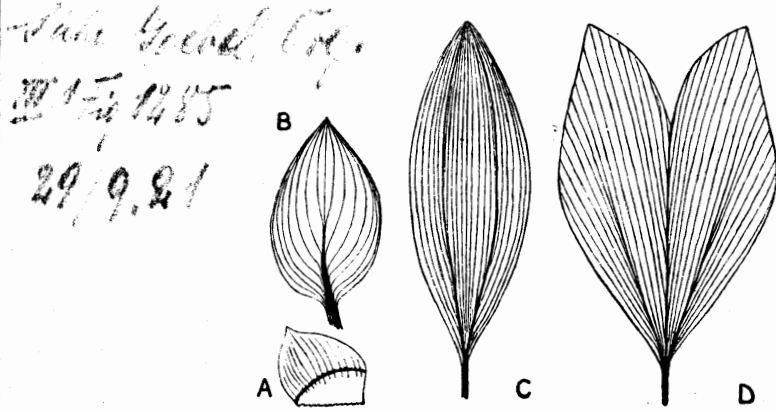

Fig. 10. $A$ junges Blatt von Iris. $B$ junges Blatt von Funkia ovata. $C$ Verlauf der Blattrippen bei Cyclanthus bipartitus. $D$ bei Carludovica Goebelii. $A$. und $B$ nach D e in eg a, $C$ und $D$ nach der Natur. diese Zusammenfassung sämtlicher Blattnerven zur Mittelrippe bereits nahe der Blattspitze einsetzt. Hierdurch und durch das fast rechtwinklige Abstehen der einzelnen Segmente vor der Mittelrippe wird eine fast monopodiale Gliederung der Blattnervatur vorgetäuscht. Jugendformen, untersucht von Phoenix und Cocos, bei deren Blättern ja auch die Segmentierung noch nicht auftritt, zeigen reinen Monokotylentypus.

Gleichfalls von dem Monokotylentyp abzuleiten sind auch die Blattformen der Cyclanthaceen. Ludovia mit einer Mittelrippe im Blatte stellt eine Parallelform zu Curculigo dar, bei Cyclanthus (Fig. 10 C) erhalten, ohne daß es zur Bildung einer Mittelrippe käme, die zwei Hälften des Blattes je eine kräftige Rippe, indem deren mittlere Nerven sich im unteren Teile jeder Blatthälfte vereinigen. Carludovica (Fig. $10 \mathrm{D}$ ) schließlich stellt eine Kombination zwischen Ludovia und dem Cyclanthustyp dar. Die Mittelrippe des Ludoviablattes bleibt erhalten und neben der eigentlichen Mittelrippe erhalten sich im basalen Teile des Blattes auch noch die für Cyclanthus charakteristischen Mittelrippen jeder Blatthälfte.

Es ist mir eine angenehme Pflicht, meinem sehr verehrten Lehrer und Chef, Herrn Geheimen Rat von Goebel, am Schlusse der Arbeit herzlich zu danken für die mir gütig gewährte Hilfe und für das`mir in freigebiger Weise zur Verfügung gestellte Pflanzenmaterial. 


\section{Literatur.}

Deinega, V., Beiträge zur Kenntnis der Entwicklungsgeschichte des Blattes und der Anlage der Gefäßbündel. Flora 1898, Bd. LXXXV.

Eichler, A. W., Zur Entwicklungsgeschichte der Palmenblätter. Abhandl. d. K. preuß. Akad. d. Wiss. zu Berlin 1885.

Goebel, K., Vergleichende Entwicklungsgeschichte der Pflanzenorgane 1883.

Ders., Organographie der Pflanzen. Spezieller Teil. 1. Aufl., 1901.

Hofmeister, W., Allgemeine Morphologie der Gewächse 1868.

Karsten, H., Die Vegetationsorgane der Palmen, eine vergleichend - anatomischphysiologische Untersuchung. Abhandl. d. K. Akad. d. Wiss. zu Berlin 1847.

Mohl, H., Über den Bau des Palmstammes. Gesammelte Schriften botanischen Inhalts XI, 1845.

Naumann, A., Zur Entwicklungsgeschichte der Palmenblätter. Flora 1887. 